\title{
A KRULL-SCHMIDT THEOREM FOR INFINITE SUMS OF MODULES
}

R. B. WARFIELD, JR.

In this note we prove a strengthened form of the well-known KrullSchmidt-Azumaya theorem [2], using results of Crawley and Jónsson [3]. We apply this result to obtain a number of results on direct sums of modules, including generalizations of Kaplansky's theorem [4] that a projective module over a logical ring is free.

1. The exchange property. A module $M$ (over an associative ring $R$ with 1 ) has the exchange property if for any module $G$, if

$$
G=M^{\prime} \oplus C=\oplus_{i \in I} D_{i}
$$

with $M^{\prime} \cong M$, then there are submodules $D_{i}^{\prime} \subseteq D_{i}$ such that $G=M^{\prime} \oplus\left(\oplus_{i \in I} D_{i}^{\prime}\right)$. Crawley and Jónsson proved $([3$, Theorem 7.1] or [6, Theorem 7]) that if $G$ is a direct sum of countably generated modules, each with the exchange property, then any two direct sum decompositions of $G$ have isomorphic refinements. If $G$ is also a direct sum of indecomposable modules, $G=\bigoplus_{i \in I} M_{i}$, then we can conclude that any direct sum decomposition of $G$ refines into a decomposition isomorphic to this one, and, in particular, any summand of $G$ is also isomorphic to a direct sum of indecomposable modules, each isomorphic to one of the $M_{i}$. In [3], this result is proved in the context of the theory of general algebraic systems, while in [6] a version in Abelian categories is proved. Our first result characterizes the indecomposable modules which have the exchange property.

Proposition 1. An indecomposable module has the exchange property if and only if its endomorphism ring is local.

Proof. An elementary argument shows that we need only consider the case where the index set $I$ is finite (see the reduction in [3, Lemma 5.1]). In this case we may actually assume that $I$ has only two elements. Suppose, then, that $\operatorname{End}(M)$ is a local ring and that $G=M \oplus C=D \oplus E$. We show there are submodules $D^{\prime}, E^{\prime}$ of $D$ and $E$ with $G=M \oplus D^{\prime} \oplus E^{\prime}$. Let the projections to $D$ and $E$ (restricted to $M$ ) be $\theta_{1}$ and $\theta_{2}$, let the projection to $M$ be $\pi$, and let the natural injections of $M, D$ and $E$ into $G$ be $\phi_{M}, \phi_{1}$, and $\phi_{2}$. Then $1_{M}=\pi \phi_{1} \theta_{1}$ $+\pi \phi_{2} \theta_{2}$ and since $\operatorname{End}(M)$ is local, one of these, say $\pi \phi_{1} \theta_{1}$, must be an automorphism. Let $\sigma$ be the inverse of this automorphism. The endo-

Received by the editors December 19, 1968. 
morphism $\eta=\phi_{1} \theta_{1} \sigma \pi$ of $G$ is a projection, whose image $N$ can be identified with the image of $\theta_{1}$. If $D^{\prime}=D \cap \operatorname{ker}(\eta)$, then $G=N \oplus D^{\prime} \oplus E$. In this decomposition, the projection onto $N$ is $\eta^{\prime} \pi_{D}$, where $\pi_{D}$ is the original projection onto $D$ and $\eta^{\prime}$ is the restriction of $\eta$ to $D$. Computation shows that $\eta^{\prime} \pi_{D} \phi_{M}$ gives an isomorphism of $M$ onto $N$, and this shows that $G=M \oplus D^{\prime} \oplus E$.

Conversely, let $M$ be an indecomposable module and suppose that $\operatorname{End}(M)$ is not a local ring. We will show that $M$ does not have the exchange property. Since the endomorphism ring is not local, there are two endomorphisms $f$ and $g$ which are not automorphisms, such that $1_{M}=f-g$. Let $A=M_{1} \oplus M_{2}$, where $M_{1} \cong M_{2} \cong M$, with projections $\pi_{1}, \pi_{2}$. We imbed $M$ in $M_{1} \oplus M_{2}$ by the homomorphism $(f, g)$ : $M \rightarrow M_{1} \oplus M_{2}$, and we call the image $M^{\prime}$. We also can imbed $M$ in $A$ by the diagonal map $\left(1_{M}, 1_{M}\right)$, and we call the image of this map $d(M)$. A standard computation shows that $A=M^{\prime} \oplus d(M)$. If the exchange property were to hold for $M$, we would have either $A=M_{1} \oplus M^{\prime}$ or $A=M_{2} \oplus M^{\prime}$, since $M_{1}$ and $M_{2}$ are indecomposable. In the first case, this would mean that $\pi_{2}(f, g)$ was an isomorphism. This is impossible since it would imply that $g$ was an automorphism. The second alternative is similarly impossible, so $M$ does not have the exchange property.

THEOREM 1. If $M$ is a direct sum of indecomposable modules, $M=\oplus_{i \in I} M_{i}$, where each $M_{i}$ is countably generated and has local endomorphism ring, then any other direct sum decomposition of $M$ refines to a decomposition isomorphic to this one, and (in particular) any summand of $M$ is again a direct sum of modules, each isomorphic to one of the original summands $M_{i}$.

This result is closely related to the well-known theorem of Azumaya [2] which says that if a module $M$ is a direct sum of indecomposable modules, $M=\oplus_{i \in I} M_{i}$, where each $M_{i}$ has local endomorphism ring, then any indecomposable summand of $M$ is isomorphic to one of the $M_{i}$ and any two decompositions of $M$ into indecomposable summands are isomorphic. A similar result was obtained for sums of indecomposable algebras with the exchange property by Crawley and Jónsson, [3, Theorem 5.3]. Proposition 3 below is an example of a result which follows from Theorem 1 but not from the Azumaya theorem.

If we restrict the number of summands to be finite, then Proposition 1 and Theorem 1 generalize to any Abelian category, as does the Azumaya theorem. See [5] for another proof and an application, and [1] for a special case and another application. For infinite sums 
we need to assume that the category satisfies axiom A6-5, in which case Proposition 1 and the Azumaya theorem carry over immediately. Theorem 1 is also valid if a suitable substitute for the countability hypothesis is provided. We refer to [6, Theorem 7] for details. The following application of Proposition 1 is also valid in any Abelian category.

Proposition 2 (Cancellation Property). Let $M$ be a module with local endomorphism ring and suppose $A$ and $B$ are modules such that $A \oplus M \cong B \oplus M$. Then $A \cong B$.

Proof. Suppose $G=A \oplus M=B \oplus M^{\prime}$, with $M^{\prime} \cong M$. Then applying the exchange property for $M$, either $G=M \oplus B$ (so $B \cong A$ trivially) or $G=M \oplus B^{\prime} \oplus M^{\prime}$, with $B^{\prime} \subseteq B$. In this case $A \cong B^{\prime} \oplus M^{\prime}$ and $B \cong B^{\prime} \oplus M$, and the result is established.

\section{Applications.}

Proposition 3. If $M$ is a module over a local ring $R$ and $M$ is a summand of a module $N$ where $N=\oplus_{i \in I} C_{i}$, and each $C_{i}$ is of the form $R / I_{i}$, where $I_{i}$ is a two-sided ideal, then $M$ is also a direct sum of cyclic modules, each isomorphic to one of the $C_{i}$.

This includes as a special case the theorem of Kaplansky [4] that a projective module over a local ring is free. It also shows that a summand of a direct sum of cyclic modules over a commutative local ring is again a direct sum of cyclic modules.

For an example in Abelian group theory, we consider torsion-free groups divisible by all primes except a given prime $p$, or equivalently, modules over the ring $R_{p}$ of rational numbers which can be written as a fraction with denominator prime to $p$. If $M$ is a torsion-free $R_{p}$-module with no divisible summand and if $M / p M \cong Z / p Z$, then $\hat{M}$ (the $p$-adic completion of $M$ ) is isomorphic to $\hat{R}_{p}$ - the additive group of $p$-adic integers-so $M$ can be regarded as a submodule of $\hat{R}_{p}$. This natural imbedding actually makes $M$ a pure submodule, so that if $x \in \hat{R}_{p}$ and $p x \in M$, then $x \in M$. Also, $E=\operatorname{End}(M)$ is thereby imbedded as a pure subring of $\hat{R}_{p}$. Assume that $M$ has finite rank, that $K$ is the quotient field of $\hat{R}_{p}$ and $L$ is the $Q$-subspace spanned by $E$. Then $L$ is a finite-dimensional integral domain and hence a field, so if $x \in E$ has an inverse in $\hat{R}_{p}$, its inverse lies in $\hat{R}_{p} \cap L=E$. Hence $E$ is a discrete valuation ring (in particular a local ring) whose maximal ideal is generated by multiplication by $p$. Theorem 1 then implies the following: 
Proposition 4. If $M$ is a torsion-free Abelian group with no divisible summand, which is divisible by all primes except one, namely $p$, and if $M$ is a direct sum of finite rank groups, $M=\bigoplus_{i \in I} M_{i}$, where $M_{i} / p M_{i} \cong Z / p Z$, then any summand of $M$ is again a direct sum of subgroups isomorphic to the $M_{i}$ and any direct sum decomposition of $M$ refines into one isomorphic to the original one.

Suppose that $R$ is any associative ring with $1, M$ is an $R$-module satisfying both the ascending and descending chain conditions (a.c.c. and d.c.c.) and $f$ is an endomorphism of $M$. Then $M=R \oplus H$, where $R$ is the set of $x \in M$ such that $f^{n}(x)=0$ for some $n$, and $H$ is the set of $x \in M$ in the image of $f^{n}$ for all $n$ (Fitting's lemma). Further, $f$ restricted to $H$ is an automorphism of $H$. From this it follows that if $M$ is indecomposable then $\operatorname{End}(M)$ is a local ring and its maximal ideal is the set of nilpotent endomorphisms. These facts can be extended as follows:

Lemma. Let $M$ be an $R$-module and $M_{i}$ a family of fully invariant submodules, and $f$ an endomorphism of $M$.

(a) If the $M_{i}$ satisfy both chain conditions and $M$ is the union of the $M_{i}$, then $M=R \oplus H$ where $R$ is the set of $x \in M$ such that $f^{n}(x)=0$ for some $n$, and $H$ is the set of $x \in M$ for which there is an i such that $x \in f^{n}\left(M_{i}\right)$ for all $n$.

(b) If the $M / M_{i}$ satisfy both chain conditions and $M=\operatorname{proj} \lim M / M_{i}$ then $M=R \oplus H$ where $R$ is the set of $x \in M$ such that for each $i$ there $i s$ an $n$ with $f^{n}(x) \in M_{i}$, and $H$ is the set of $x \in M$ in the image of $f^{n}$ for all $n$.

In both cases $f$ restricts to an automorphism of $H$. In particular, if in either of these cases $M$ is indecomposable, then $\operatorname{End}(M)$ is a local ring.

Proposition 5. Let $R$ be a ring which is either left Noetherian or commutative and $M$ a left $R$-module which is a direct sum of submodules satisfying the d.c.c. By a further decomposition, these summands may be assumed indecomposable. Then any summand of $M$ is also a direct sum of submodules satisfying the d.c.c. and any direct sum decomposition of $M$ refines into a decomposition isomorphic to the original one.

Proof. It suffices to show that over such a ring an indecomposable module $N$ with d.c.c. satisfies condition (a) of the lemma. Let $N_{1}$ be the socle of $N$ and $N_{n+1}$ the inverse image in $N$ of the socle of $N / N_{n}$. We claim that $N$ is the union of the submodules $N_{n}$, and to show this it suffices to remark that any cyclic $R$-module satisfying the d.c.c. also satisfies the a.c.c., so that for $x \in N, R x \subseteq N_{n}$ for some $n$. 
Proposition 6. Let $R$ be a left-Noetherian ring and $I$ a two-sided ideal generated by a finite number of elements in the center of $R$, such that $\bigcap_{n>0} I^{n}=0, R$ is complete in the $I$-adic topology, and $R / I$ satisfies the d.c.c. Then if $M$ is a left $R$-module which is a direct sum of finitely generated modules, any summand of $M$ is again a direct sum of finitely generated modules. Further, any direct sum decomposition of $M$ refines into a direct sum of indecomposable, finitely generated modules, and this decomposition is unique up to isomorphism.

Proof. Clearly if $R / I$ satisfies the d.c.c., so does $R / I^{n}$ since $R$ is left-Noetherian. Hence if $N$ is finitely generated, $N / I^{n} N$ satisfies both chain conditions. Therefore it suffices to show that if $N$ is a finitely generated $R$-module, then $\bigcap_{n>0} I^{n} N=0$, and $N$ is complete in the $I$-adic topology. This actually holds without the hypothesis that $R / I$ satisfies the d.c.c. Clearly a finitely generated module $L$ with $\bigcap_{n>0} I^{n} L=0$ is complete in the $I$-adic topology. We represent $N$ as a quotient, $N=F / K$, where $F$ is free and finitely generated. It is enough to show that $\bigcap_{n>0} I^{n} N=0$, and since $\bigcap_{n>0} I^{n} N$ is just the closure of 0 in the $I$-adic topology, this is equivalent to showing that $K$ is a closed submodule in the topology of $F$. Clearly $\bigcap_{n>0} I^{n} K=0$, so $K$ is complete, and we need only show that the $I$-adic topology on $K$ agrees with the topology induced from the $I$-adic topology on $F$. This is a standard argument in commutative algebra (Bourbaki, Algèbre commutative, Chapter III, Corollary $1, \mathrm{p}$. 61) where $R$ is assumed to be commutative, and the fact that $I$ is generated by elements of the center of $R$ is enough to assure that the same arguments are valid in this case.

CoRollary. If $P$ is a projective module over a ring satisfying the hypotheses of Proposition 6, then $P$ is a direct sum of indecomposable left ideals generated by idempotents and any two such decompositions of $P$ are isomorphic.

For examples, we remark that an Artinian ring satisfies these hypotheses trivially, and so does the group ring of a finite group over the $p$-adic integers.

\section{BIBLIOGRAPHY}

1. M. Atiyah, On the Krull-Schmidt theorem with application to sheaves, Bull. Soc. Math. France 84 (1956), 307-317.

2. G. Azumaya, Corrections and supplementaries to my paper concerning KrullRemak-Schmidt's theorem, Nagoya Math. J. 1 (1950), 117-124.

3. P. Crawley and B. Jónsson, Refinements for infinite direct decompositions of algebraic systems, Pacific J. Math. 14 (1964), 797-855. 
4. I. Kaplansky, Projective modules, Ann. of Math. 68 (1958), 372-377.

5. E. Walker, Quotient categories and quasi-isomorphisms of Abelian groups, Proc. Colloq. Abelian Groups (Tihany), Akad. Kiad6, Budapest, 1964, pp. 147-162.

6. R. B. Warfield, Jr., Decompositions of injective modules, Pacific J. Math. (to appear).

UNIVERSITY OF WASHINGTON 